\title{
A fourth-order cumulant orthonormal propagator rooting method based on Toeplitz approximation
}

\author{
Heping Shi ${ }^{1} \mathbb{D}$, Ning Ma², Zhiwei Guan ${ }^{1,3^{*}}$, Lizhu Zhang ${ }^{1}$ and Shan Jiang ${ }^{4}$
}

\author{
*Correspondence: zhiwguan@163. \\ com \\ ${ }^{1}$ School of Automobile and \\ Transportation, Tianjin University of \\ Technology and Education, Tianjin \\ 300222, China \\ ${ }^{3}$ Tianjin Sino-German University of \\ Applied Sciences, Tianjin 300350, \\ China \\ Full list of author information is \\ available at the end of the article
}

\begin{abstract}
A novel Toeplitz fourth-order cumulant (FOC) orthonormal propagator rooting method (TFOC - OPRM) of direction-of-arrival (DOA) estimation for uniform linear array $(U L A)$ is proposed in this paper. Specifically, the modified (i.e., reduceddimension) FOC (MFOC) matrix is achieved at first via removing the redundant information encompassed in the primary FOC matrix; then, the TFOC matrix which possesses Toeplitz structure can be recovered by utilizing the Toeplitz approximation method. To reduce the computational complexity, an effective method based on the polynomial rooting technology is adopted. Finally, the DOAs of incident signals can be estimated by exploiting orthonormal propagator rooting method. The theoretical analysis coupled with simulation results show that the proposed resultant algorithm can reduce the computational complexity significantly, as well as improve the estimation performance in both spatially white noise environment and spatially color noise environment.

Keywords: Direction-of-arrival (DOA), Fourth-order cumulants (FOC), Polynomial rooting, Toeplitz approximation, Orthonormal propagator method (OPM)
\end{abstract}

\section{Introduction}

Direction-of-arrival estimation based on antenna array is one of the important directions of research hotspot in array signal processing, which has wide prospects of application in military and civil fields such as wireless communications, radar, passive sonar, biomedicine, and seismic exploration [1-4]. Various high-resolution algorithms, such as multiple signal classification (MUSIC) algorithm [5] and estimating signal parameter via rotational invariance technique (ESPRIT) [6] approaches, have been proposed to achieve direction-of-arrival $(D O A)$ estimation of narrowband far-field signal sources. However, these subspace-based $D O A$ estimation algorithms described above are not only very sensitive to the noise, but also require the noise's characteristics of the sensors in advance. Furthermore, it is restricted that the total number of sources acting on the array must be less than or equal with sensors [7]. When the constrained condition cannot be met in practical environments, the estimation performance of those aforementioned algorithms may run into a stone wall. Fortunately, much more

(c) The Author(s). 2020 Open Access This article is licensed under a Creative Commons Attribution 4.0 International License, which permits use, sharing, adaptation, distribution and reproduction in any medium or format, as long as you give appropriate credit to the original author(s) and the source, provide a link to the Creative Commons licence, and indicate if changes were made. The images or other third party material in this article are included in the article's Creative Commons licence, unless indicated otherwise in a credit line to the material. If material is not included in the article's Creative Commons licence and your intended use is not permitted by statutory regulation or exceeds the permitted use, you will need to obtain permission directly from the copyright holder. To view a copy of this licence, visit http://creativecommons.org/licenses/by/4.0/. 
attentions have been paid to this issue, and much more efforts have been made to overcome the above drawbacks. Motivated by the truth that the high-order cumulant-based $(H O C)$ is asymptotically insensitive to Gaussian noise, which can be recognized as a promising technique for direction finding by adopting sensor array [8-10]. Besides, another key motivation of using $\mathrm{HOC}$ is the ability to resolve more number of sources than or equal to that of the array elements [11]. However, the process of eigenvalue decomposition (EVD) or singular value decomposition (SVD) requires large amount of calculation and time taken, which greatly affects the development of rapid source location. Marcos and co-workers [12, 13] firstly proposed so-named propagator method (PM) to obtain the signal and noise subspaces by executing a linear-partition operation, which can decrease the computational complexity effectively. Specifically, the algorithm under the conditions of medium and high signal-to-noise ratio can achieve the same performance as that of the traditional high-resolution algorithms but with higher calculation efficiency. Base on [12, 13], numerous modifications of PM have been proposed, such as $[14,15]$, to achieve the low-complexity $D O A$ estimation. In [16], an efficient HOPM algorithm was proposed by making full use of intrinsic multi-dimensional characteristics and affordable computability. AFOC - based and OPM - like (FOC - OPM) algorithm [17] was proposed to gain good location performance. However, the computational complexity of this method is high due to a great number of redundant information still existing in the $F O C$ matrix. To mitigate this shortcoming, the improved FOC algorithm [18] was proposed to reduce the computational complexity. However, the performance of the algorithm cannot be asymptotically optimal due to the estimation error of the FOC matrix. Zhang et al. [19] derived a root-MUSIC method using a co-prime linear array to improve the estimation accuracy with low complexity. In [20-22], a similar polynomial root-based method was chosen to realize low-complexity for $D O A$ estimation. In [23-28], the fractal structure modeling based was utilized to improve the performance of the fractal antennas. In $[29,30]$, the wavelet analysis-based method was proposed to deal with application in signal processing.

In this paper, a novel TFOC-OPRM algorithm is introduced. The contributions of this paper are twofold: Firstly, the reduced dimension matrix is obtained to reduce the computational complexity by removing a large number of redundant elements from the original FOC matrix while maintaining the effective aperture of the virtual array in unchanged state. Secondly, the Toeplitz structure is recovered by the Toeplitz operation of the reduced dimension FOC matrix, and the DOA estimation of the recovered Toeplitz structure matrix is performed based on the polynomial root method.

\section{Data model}

Consider $M$ narrowband far-field sources $s_{i}(t),(i=1, \cdots, M)$ impinging on a uniform linear array $(U L A)$ with $N$ equispaced omnidirectional sensors, where the distance between adjacent sensors is equal to half the wavelength. Assume that the incoming sources are stationary and mutually independent. The noise is the additive white/color Gaussian one and statistically independent of the sources. Let the first sensor be the reference, and then, the observed data received in time $t$ at the $k$ th sensor can be expressed as 


$$
x_{k}(t)=\sum_{i=1}^{M} a_{k}\left(\theta_{i}\right) s_{i}(t)+n_{k}(t), \quad k=1, \cdots, N
$$

where $s_{i}(t)$ is the $i$ th source, $n_{k}(t)$ is the Gaussian noise at the $k$ th sensor, and $a_{k}\left(\theta_{i}\right)$ is the response of the $k$ th sensor corresponding to the $i$ th source and can be expressed as

$$
a_{k}\left(\theta_{i}\right)=\exp \left(j 2 \pi(d / \lambda) k \sin \theta_{i}\right)
$$

where $\lambda$ is the central wavelength and $d$ is the spacing between two adjacent sensors. Therefore, the matrix form of (1) can be expressed as

$$
\mathbf{X}(t)=\mathbf{A S}(t)+\mathbf{N}(t)
$$

where $\mathbf{X}(t)=\left[x_{1}(t), \cdots, x_{N}(t)\right]^{T}$ is the $N \times 1$ received source vector, $\mathbf{S}(t)=\left[s_{1}(t), \cdots, s_{M}(t)\right]^{T}$ is the $M \times 1$ radiating source vector, $\mathbf{A}=\left[a\left(\theta_{1}\right), \cdots, a\left(\theta_{M}\right)\right]$ is the $N \times M$ array manifold matrix, and $\mathbf{N}(t)=\left[n_{1}(t), \cdots, n_{N}(t)\right]^{T}$ denotes the $N \times 1$ complex Gaussian noise vector.

Assuming that the source signals are zero-mean stationary random process, the $F O C$ can be defined as

$$
\begin{array}{r}
\operatorname{cum}\left(k_{1}, k_{2}, k_{3}^{*}, k_{4}^{*}\right)=E\left(x_{k_{1}}(t) x_{k_{2}}(t) x_{k_{3}}^{*}(t) x_{k_{4}}^{*}(t)\right)- \\
E\left(x_{k_{1}}(t) x_{k_{3}}^{*}(t)\right) E\left(x_{k_{2}}(t) x_{k_{4}}^{*}(t)\right)- \\
E\left(x_{k_{1}}(t) x_{k_{4}}^{*}(t)\right) E\left(x_{k_{2}}(t) x_{k_{3}}^{*}(t)\right)- \\
E\left(x_{k_{1}}(t) x_{k_{2}}(t)\right) E\left(x_{k_{3}}^{*}(t) x_{k_{4}}^{*}(t)\right) \\
k_{1}, k_{2}, k_{3}, k_{4} \in[1, \cdots, N]
\end{array}
$$

where $x_{k_{m}}(m=1,2,3,4)$ is the stochastic process. Apparently, $\operatorname{cum}\left(k_{1}, k_{2}, k_{3}^{*}, k_{4}^{*}\right)$ has $N^{4}$ values with the change of $k_{1}, k_{2}, k_{3}, k_{4}$. For simplicity, Eq. (4) can be written in matrix form, which is denoted by cumulant matrix $\mathbf{C}_{4}$, and $\operatorname{cum}\left(k_{1}, k_{2}, k_{3}^{*}, k_{4}^{*}\right)$ appears as the $\left[\left(k_{1}-1\right) N+k_{2}\right]$ th row and $\left[\left(k_{3}-1\right) N+k_{4}\right]$ th column of $\mathbf{C}_{4}$.

$$
\begin{aligned}
& \mathbf{C}_{4}\left[\left(k_{1}-1\right) N+k_{2},\left(k_{3}-1\right) N+k_{4}\right] \\
& =\operatorname{cum}\left(k_{1}, k_{2}, k_{3}^{*}, k_{4}^{*}\right) \\
& =\mathbf{B C}_{s} \mathbf{B}^{\mathrm{H}}
\end{aligned}
$$

where $\mathbf{B}$ and $\mathbf{C}_{S}$ represent the extended array manifold and the $F O C$ matrix of incident source signals, respectively. $\mathbf{B}=\mathbf{A} \otimes \mathbf{A}$ and each column of $\mathbf{B}$ is $\mathrm{b}(\theta)=\boldsymbol{a}(\theta) \otimes \boldsymbol{a}(\theta)$. It is obvious that $\mathrm{b}(\theta)$ is a $N^{2} \times 1$ vector, which means that the array aperture of ULA is extended. That is, the number of resolved source signals is no less than that of sensors.

\section{The proposed method}

\subsection{The effective array aperture extended}

As proven in [31], an array of $N$ arbitrary identical omnidirectional sensors can be extended to at most of $N^{2}-N+1$. Especially, the number of virtual elements is $2 N-1$ for ULA according to [31]. In order to discuss the effective aperture of ULA, four real elements $(N=4)$ are considered, and $\mathrm{b}(\theta)$ can be expressed in detail as follows: 


$$
\begin{aligned}
\mathbf{b}(\theta)= & a(\theta) \otimes a(\theta) \\
= & {\left[1, z, z^{2}, z^{3}, z, z^{2}, z^{3}, z^{4},\right.} \\
& \left.z^{2}, z^{3}, z^{4}, z^{5}, z^{3}, z^{4}, z^{5}, z^{6}\right]^{\mathrm{T}}
\end{aligned}
$$

where $z=\exp (j 2 \pi(d / \lambda) \sin \theta)$. Equation (6) shows that there is a lot of redundancy in expanded steering vector $\mathrm{b}(\theta)$. That is, only from 1 st to $N$ th and all $k N$ th $(k=2, \cdots, N)$ items of the $\mathrm{b}(\theta)$ are valid, while others are redundant ones. To eliminate these repetitive elements, a $(2 N-1) \times(2 N-1)$ matrix $\mathbf{R}_{4}$ is defined firstly. Next, the 1 st to $N$ th and all $k N$ th $(k=2, \cdots, N)$ rows of $\mathbf{C}_{4}$ are taken out in sequence, and then these rows are stored in the 1st to $(2 N-1)$ th row of the new matrix $\mathbf{R}_{4}$. The same operation is performed on the 1st to $N$ th and all $k N$ th $(k=2, \cdots, N)$ columns of $\mathbf{C}_{4}$ to obtain the 1st to $(2 N-1)$ th columns of $\mathbf{R}_{4}$. Similar to Eq. (5), $\mathbf{R}_{4}$ can be expressed as

$$
\mathbf{R}_{4}=\mathbf{D C}_{s} \mathbf{D}^{\mathrm{H}}
$$

where $\mathbf{D}$ denotes the extended array manifold without redundancy, and each column of D has the form of $\mathbf{d}(\theta)=\left[1, \cdots, z^{2 N-2}\right]^{T}$. Therefore, the reduced-dimension $\mathbf{R}_{4}$ not only contains all of the information about original matrix $\mathbf{C}_{4}$, but also keeps the extended array aperture unchanged.

\subsection{The TFOC-OPRM method}

When the incident targets are considered as statistically independent signal sources, the ideal $\mathbf{R}_{4}$ has a Toeplitz structure. However, in practical applications, for example, due to finite sampling snapshots and the low SNR, the matrix $\mathbf{R}_{4}$ obtained at this time does not meet the Toeplitz structure anymore; instead, it becomes a diagonally dominant matrix. The happening of such condition will have a negative impact on the performance of the final DOA estimation. In order to improve the DOA estimation accuracy of the antenna array, the first task is to recover the Toeplitz structure of matrix $\hat{\mathbf{R}}_{4}$, that is, to get Toeplitz matrix $\hat{\mathbf{R}}_{4 \mathrm{~T}}$. Then, a $\mathbf{R}_{4 \mathrm{~T}}$ of Toeplitz matrix can be approached to the real reduced dimension by solving the following optimization problem:

$$
\min _{\mathbf{R}_{4 \mathrm{~T}} \in \mathbf{S}_{\mathrm{T}}}\left\|\mathbf{R}_{4 \mathrm{~T}}-\mathbf{R}_{4}\right\|
$$

where $\mathbf{S}_{\mathrm{T}}$ represents Toeplitz matrices, and the entries of the Toeplitz matrix $\mathbf{R}_{4 \mathrm{~T}}$ can be written as

$$
\gamma_{h}=(2 N-1-h+1)^{-1} \sum_{p=1}^{2 N-1-h+1} r_{p(p+h-1)}
$$

where the element $r_{p(p+h-1)}$ denotes the $p$ th row and $(p+h-1)$ th column of $\mathbf{R}_{4}$, $h \in[1, \cdots, 2 N-1]$. And then $\mathbf{R}_{4 \mathrm{~T}}$ can be obtained by the following Toeplitization operator:

$$
\mathbf{R}_{4 \mathrm{~T}}=\operatorname{Toep}\left(\gamma_{1}, \ldots . . \gamma_{2 N-1}\right)
$$

where Toep stands for the Toeplitization operator.

Although conventional algorithms, such as MUSIC and ESPRIT, can be applied to estimateDOAs based on the $\mathbf{R}_{4 \mathrm{~T}}$, the computational burden is much heavier due to the 
EVD and SVDinvolved. Therefore, we apply OPM for estimating the DOAs to reduce the complex computations effectively.

The presented propagator method is based on the following partition

$$
\mathbf{R}_{4 \mathrm{~T}}=\left[\begin{array}{l}
\mathbf{R}_{4 \mathrm{~T} 1} \\
\mathbf{R}_{4 \mathrm{~T} 2}
\end{array}\right]
$$

where the dimensions of $\mathbf{R}_{4 \mathrm{~T} 1}$ and $\mathbf{R}_{4 \mathrm{~T} 2}$ are $M \times(2 N-1)$ and $(2 N-1-M) \times(2 N-1)$, respectively. The $M \times(2 N-1-M)$ propagator matrix $\mathbf{P}$ is defined as a unique linear operator which satisfies the following condition

$$
\mathbf{P}^{\mathrm{H}} \mathbf{R}_{4 \mathrm{~T} 1}=\mathbf{R}_{4 \mathrm{~T} 2}
$$

Define $\mathbf{Q}^{\mathrm{H}}=\left[\begin{array}{ll}\mathbf{P}^{\mathrm{H}} & -\mathbf{I}_{2 N-1-M}\end{array}\right]$, and combine with equation (11)

$$
\begin{aligned}
\mathbf{Q}^{\mathrm{H}} \mathbf{R}_{4 \mathrm{~T}}= & {\left[\begin{array}{ll}
\mathbf{P}^{\mathrm{H}} & -\mathbf{I}_{2 N-1-M}
\end{array}\right]\left[\begin{array}{l}
\mathbf{R}_{4 \mathrm{~T} 1} \\
\mathbf{R}_{4 \mathrm{~T} 2}
\end{array}\right] } \\
& =\mathbf{0}_{(2 N-1-M) \times(2 N-1)}
\end{aligned}
$$

Equation (13) shows that the $\mathbf{R}_{4 \mathrm{~T}}$ is orthogonal to the columns of $\mathbf{Q}^{\mathrm{H}}$, and the propagator matrix $\mathbf{P}$ can be obtained by minimizing the cost function $\xi(\mathbf{P})$

$$
\xi(\mathbf{P})=\left\|\mathbf{R}_{4 \mathrm{~T} 2}-\mathbf{P}^{\mathrm{H}} \mathbf{R}_{4 \mathrm{~T} 1}\right\|_{\mathrm{F}}^{2}
$$

where $\|\bullet\|_{F}$ indicates the Frobenius norm, and the optimal solution $\mathbf{P}$ is given by

$$
\mathbf{P}=\left(\mathbf{R}_{4 \mathrm{~T} 1} \mathbf{R}_{4 \mathrm{~T} 1}^{\mathrm{H}}\right)^{-1} \mathbf{R}_{4 \mathrm{~T} 1} \mathbf{R}_{4 \mathrm{~T} 2}^{\mathrm{H}}
$$

In order to introduce the orthonormalization, the orthonormalized matrix $\mathbf{Q}_{0}$ is obtained as follows

$$
\mathbf{Q}_{0}=\mathbf{Q}\left(\mathbf{Q}^{\mathrm{H}} \mathbf{Q}\right)^{-1 / 2}
$$

Therefore, the following spectral function $p(\theta)$ can be formed to estimate the DOAs of source signals

$$
P(\theta)=\frac{1}{\boldsymbol{d}^{\mathrm{H}}(\theta) \mathbf{Q}_{0} \mathbf{Q}_{0}{ }^{\mathrm{H}} \boldsymbol{d}(\theta)}
$$

It can be seen from function (17) that the MDOAs of the incoming signals can be obtained by means of one-dimensional $(1-\mathrm{D})$ spectrum-peak search over $\theta$. However, to further reduce the computational burden, we can improve function (17) to derive a more efficient search-free modification estimator in computation based on polynomial rooting [32]. In order to further reduce the computational complexity of the algorithm, the method based on polynomial roots is used to improve the spatial spectrum estimation function, so as to obtain more efficient estimators in the calculation, with the specific description of the algorithm given as follows.

Set $z=\exp (j 2 \pi(d / \lambda) \sin \theta)$, we have $\mathbf{d}=\mathbf{d}(z)$

$$
\mathbf{d}(z)=\left[1, \ldots, z^{2 N-2}\right]^{T}
$$

Then, the denominator of the estimator (17) can be re-expressed with the following polynomial format 


$$
f(z)=\boldsymbol{d}^{\mathrm{H}}(z) \mathbf{Q}_{0} \mathbf{Q}_{0}{ }^{\mathrm{H}} \boldsymbol{d}(z)
$$

In an ideal condition, there should be exactly $M$ numbers of roots, that is, $z_{1}, z_{2}, \cdots$, $z_{M}$ distributing over the unit circle, and these $M$ numbers of roots are exactly the roots of the polynomial $f(z)$. However, in practical application, due to the influence of various complex factors in the environment, the $M$ roots of the equation $f(z)$ cannot be strictly distributed on the unit circle. In this case, only $M$ roots close to the unit circle need to be selected, similarly to the Root - MUSIC approach [32-34]. After $M$ roots $\left\{z_{1}, \cdots, z_{i}\right.$, $\left.\cdots z_{M}\right\}$ are obtained, the DOA estimation of the incident target signal source can then be completed by the following formula

$$
\theta_{i}=\arcsin \left(\frac{\lambda}{2 \pi d} \operatorname{angle}\left(z_{i}\right)\right) \quad i=1, \ldots, M
$$

So far as it is concerned, the specific operational steps of the proposed Toeplitz fourth-order cumulant orthogonal propagation method based on polynomial roots under limited sampling snapshots can be summarized as follows:

Step 1 Estimate $\mathbf{C}_{4}$ from the received data by (5).

Step 2 Obtain the dimension reduction matrix $\mathbf{R}_{4}$ by removing the redundant items from the expanded matrix $\mathbf{C}_{4}$.

Step 3 Reconstruct the Toeplitz matrix $\mathbf{R}_{4 \mathrm{~T}}$ by performing Toeplitz approximation on $\mathbf{R}_{4}$ as formulas (9) and (10).

Step 4 Estimate the linear operator $\mathbf{P}$ according to Eqs. (14) and (15), then calculate the standard orthonormalized matrix $\mathbf{Q}_{0}$ based on Eq. (16).

Step 5 Obtain the polynomial function (19), and further to it, obtain the $M$ roots closest to the unit circle, i.e., the roots of the $f(z)$.

Step 6 Obtain the direction estimation of the incoming wave of the incident target signal source from (20).

\subsection{Complexity analysis}

As for the analysis of computational complexity, the main parts of computation are considered, that is, the construction of the cumulant matrix, the linear operation, the spectral peak search operation, the Toeplitz operation, and the polynomial rooting operation. To further prove the superiority of the TFOC-OPRM algorithm in terms of computational complexity, FOC - OPM and MFOC - OPM are used as the comparative algorithms.

For the FOC-OPM technique, the main operation amount comes from three major parts, that is, to calculate the $N^{2} \times N^{2}$ cumulant matrix, to perform the linear operator of cumulant matrix, and to execute one spectral search. Therefore, the computational complexity of the FOC - OPM technique is $O\left(\left(9 N^{4} L\right)+\left(M N^{4}\right)+(180 / \Delta \theta) N^{4}\right)$, in which $L$ and $\Delta \theta$ denote the number of snapshots and the interval of the angular scanning, respectively. For the MFOC - OPM algorithm, the main calculation amount comes from constructing one $(2 N-1) \times(2 N-1)$ cumulant matrix, performing the linear operator of cumulant matrix, and executing one spectral search. Therefore, the computational load of the MFOC - OPM is $O\left(9(2 N-1)^{2} L+M(2 N-1)^{2}+(180 / \Delta \theta)(2 N-1)^{2}\right)$. 
For the proposed TFOC-OPRM algorithm, the major computational complexity comes from forming one $(2 N-1) \times(2 N-1)$ cumulant matrix, to perform Toeplitz operation, to perform the linear operator of cumulant matrix, and to execute once polynomial rooting operation. Therefore, the computational complexity is $O\left(9(2 N-1)^{2} L+\right.$ $\left.2(2 N-1)-1+\left(2(2 N-1)^{2}-(2 N-1)\right)+2(2 N-1)-1+M(2 N-1)^{2}+M N\right)$.

From the above analysis, it can be obviously seen that the computational complexity of TFOC-OPRM algorithm proposed in this paper is significantly lower than that of both FOC-OPM algorithm and MFOC-OPM algorithm. The main reason is that the polynomial root method has been involved to reduce the computational complexity further.

\section{Results and discussion}

In this section, the proposed TFOC-OPRM algorithm, as well as FOC-OPM [17] andMFOC - OPM [18] algorithms that are used for the purpose of comparison are simulated in the environment of spatial white noise and spatial color noise respectively to verify the superiority of the proposed algorithm. In the simulation experiment, the ULA composed of three antenna elements $(N=3)$ is used, in which the interval between adjacent antenna elements is $d=\lambda / 2$. It is assumed that there are three far-field narrow-band statistically independent target signal sources $(M=3)$, whose incident angles are $\left\{-45^{\circ}, 15^{\circ}, 40^{\circ}\right\}$ respectively, with the Gaussian white/color noise being considered. Both the proposed TFOC-OPRM algorithm and these two comparative algorithms take 500 Monte-Carlo simulations each time as their estimated performance value. Two performance indexes, namely, normalized probability of success (NPS) and estimated root-mean-square errors (RMSEs), are defined to evaluate the performance of these three algorithms. And the RMSEs and NPS are respectively expressed as

$$
\begin{aligned}
& \text { RMSEs }=\sqrt{\frac{1}{500 M} \sum_{i=1}^{500} \sum_{n=1}^{M}\left(\hat{\theta}_{n}(i)-\theta_{n}\right)^{2}} \\
& \mathrm{NPS}=\frac{\Upsilon_{\text {suc }}}{\mathrm{T}_{\text {total }}}
\end{aligned}
$$

where $\hat{\theta}_{n}(i)$ refers to the estimated value of the reference value $\theta_{n}$ in the $i$ th time Monte Carlo trial. The $\Upsilon_{\text {suc }}$ and $\mathrm{T}_{\text {total }}$ denote the times of successes and Monte Carlo trial, respectively. Furthermore, it should be noted that the defined success of a simulation experiment satisfies $\max \left(\left|\hat{\theta}_{n}-\theta_{n}\right|\right)<\varepsilon$, and $\varepsilon$ in the formula equals to 0.8 and 1.5 for experiments 2 and 3 , respectively.

\subsection{Experiment 1: the spatial spectrum estimation}

In the first experiment, the input SNR and the number of snapshots are set to be $10 \mathrm{~dB}$ and 500, respectively. Figure 1 shows the spatial spectrum of the proposed TFOC OPRM, FOC-OPM, and MFOC-OPM algorithms in both spatially white noise and spatially color noise environments. It can be observed from the curves in Fig. 1 that all of the three algorithms have successfully located the sharp peak corresponding to the incident angle. Note that the angular resolution performance of the three algorithms in spatially white noise situation provides better than that of spatially color noise situation. 


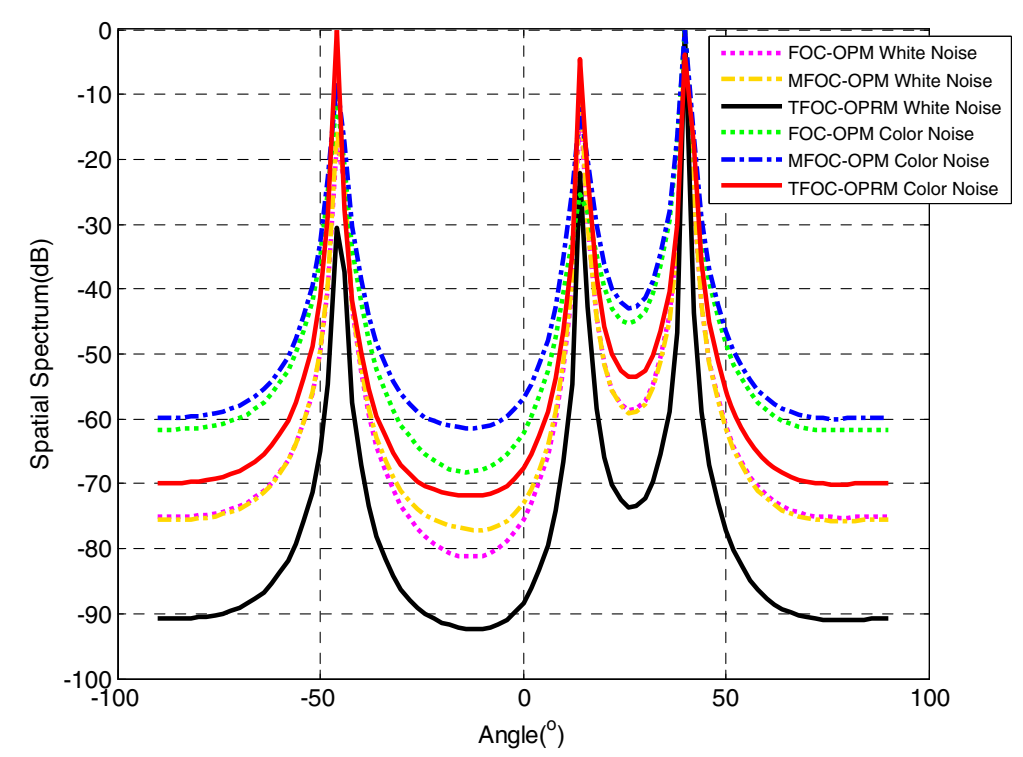

Fig. 1 Spatial spectrum of different algorithms

Further analysis indicates that no matter whether in spatially white noise situation or in spatially color noise situation, the angular resolution of the proposed TFOC - OPRM algorithm is much higher than that of both MFOC-OPM and FOC-OPM algorithms. The reason is that the proposed TFOC-OPRM algorithm can recover the Toeplitz structure of $\mathbf{R}_{4}$, making the Toeplitz matrix $\mathbf{R}_{4 \mathrm{~T}}$ closer to the real situation.

\subsection{Experiment 2: RMSEs and NPS versus SNR}

The main objective of this experiment is to evaluate the performance of the TFOC OPRM algorithm, FOC-OPM algorithm and MFOC-OPM algorithm in terms of RMSEs and NPS with the change of input SNR. The number of sampling snapshots is $L=2000$, the input SNRchanges from 8 to $24 \mathrm{~dB}$, with the step being $2 \mathrm{~dB}$. Figures 2 and 3 plot the RMSEs and NPS of DOA estimation with the proposed TFOC - OPRM algorithm and the comparison algorithms as the input SNR changes, respectively. As illustrated in Fig. 2, the RMSEs of the three algorithms decrease monotonically with the increase of the input SNR. Further analysis shows that in the environment of spatial white noise, with the increase of input SNR, the RMSEs performance curve of TFOC OPRM algorithm is better than that of FOC-OPM algorithm and that of MFOC OPM algorithm; in the environment of spatial color noise, the RMSEs performance curve of TFOC - OPRM algorithm is better than that of MFOC - OPM algorithm. That is, no matter whether in spatially white noise situation or in spatially-color noise situation, the RMSEs performance of the proposed TFOC - OPRM algorithm achieves better than that of both MFOC - OPM and FOC - OPM algorithms. In addition, when the input SNR changes between 8 and $14 \mathrm{~dB}$, the proposed TFOC - OPRM algorithm manages to achieve almost the same RMSE performance as the FOC-OPM algorithm. But when the input SNR is higher than $14 \mathrm{~dB}$, the performance of TFOC - OPRM becomes better than that of FOC - OPM. From Fig. 3, it can be concluded that the NPS performance of the proposed TFOC-OPRM algorithm is better than that of the FOC-OPM 


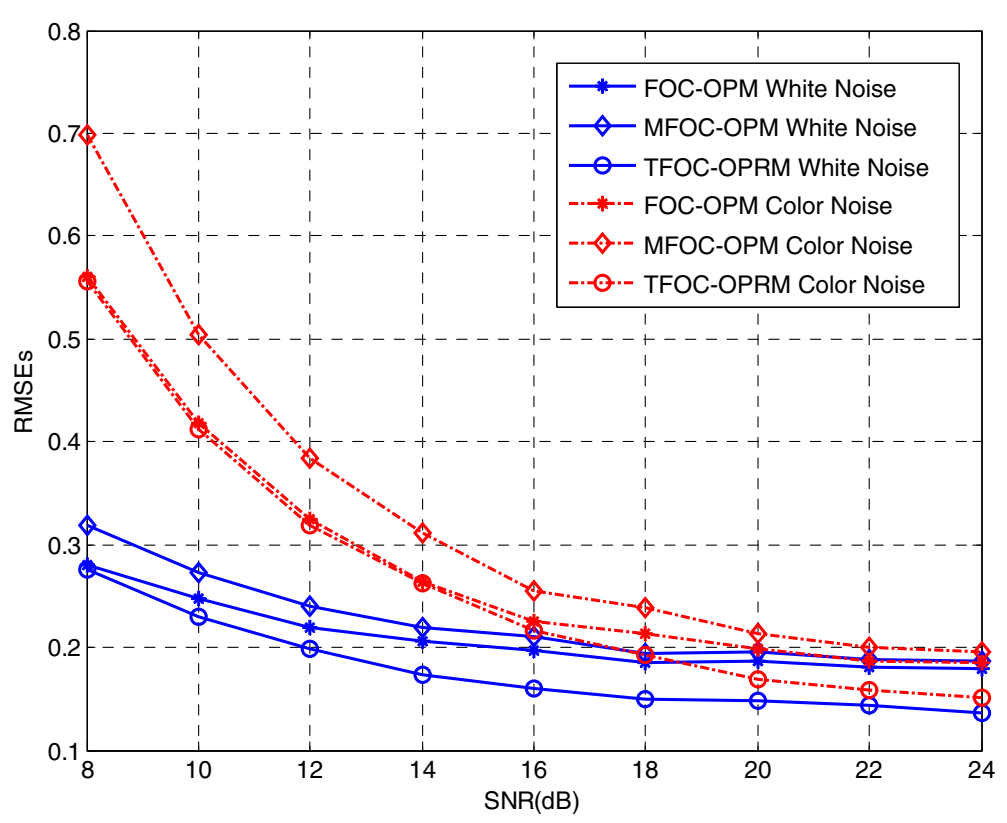

Fig. 2 RMSEs of the DOAs versus SNR

algorithm and MFOC - OPM algorithm in the case of low input SNR (between 8 and $10 \mathrm{~dB}$ ) in spatially white noise situation. With the increase of input SNR, theNPS of all of the three algorithms ultimately is 1 . It is also worth noting that when the SNR changes between 8 and $16 \mathrm{~dB}$ in spatially color noise situation, the performance of the proposed algorithm is better than that of the compared algorithms. In addition to that, the proposed algorithm not only removes a lot of redundant data in the original FOC, but also restores the Toeplitz structure of the reduced dimensional FOC. Moreover, it adopts the method of finding roots of polynomials. Therefore, the proposed TFOC -

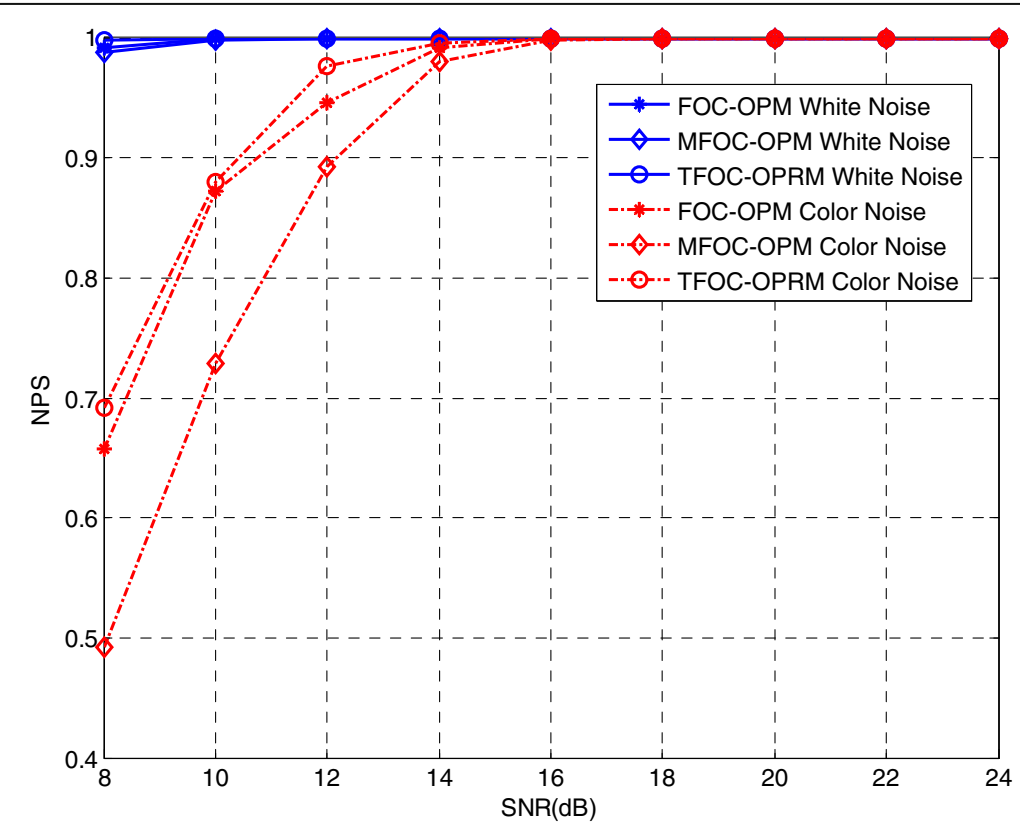

Fig. 3 NPS of the DOAs versus SNR 
OPRM algorithm not only reduces the computational complexity, but also improves the accuracy of DOA estimation.

\subsection{Experiment 3: RMSEs and NPS versus snapshots}

The main objective of this experiment is to verify the performance of the RMSEs and the NPS of TFOC-OPRM algorithm, FOC-OPM algorithm, and MFOC-OPM algorithm when the number of sampling snapshots changes under the environment of Gaussian white noise and color noise. The input SNR is set to $10 \mathrm{~dB}$, the number of sampling snapshots changes from 400 to 2000, with the step of 200. Shown in Figs. 4 and 5 are the performance curves of RMSEs and NPS of the proposed algorithm and the comparative algorithms as the number of sampling snapshots changes. It can be seen from the performance curves demonstrated in Figs. 4 and 5 that when the number of sampling snapshots varies from 400 to 1000, both RMSEs and NPS show the presence of a large degree of jitter. The main reason for this unsatisfied performance is that the number of sampling snapshots is relatively small, resulting in too little data acquired. In other words, the estimated matrix $\hat{\mathbf{R}}_{4}$ deviates greatly from the ideal matrix $\mathbf{R}_{4}$ due to the number of sampling snapshots that is relatively small. With the increasing number of sampling snapshots, we can see that the performance curves tend to be stable gradually. At the same time, it can be observed that the TFOC - OPRM algorithm proposed in this paper achieves more satisfactory estimation performance thanMFOC OPM and FOC-OPM algorithms, either in the condition of spatial-white noise or in the condition of spatial-color noise. And the estimation performance of the three algorithms in spatially-white noise situation provide better than that of spatially color noise situation. Note that the computational complexity of proposed algorithm is significantly lower than that of the FOC-OPM algorithm due to the fact that the redundant information of the original cumulant matrix is removed. Moreover, the Toeplitz approxi-

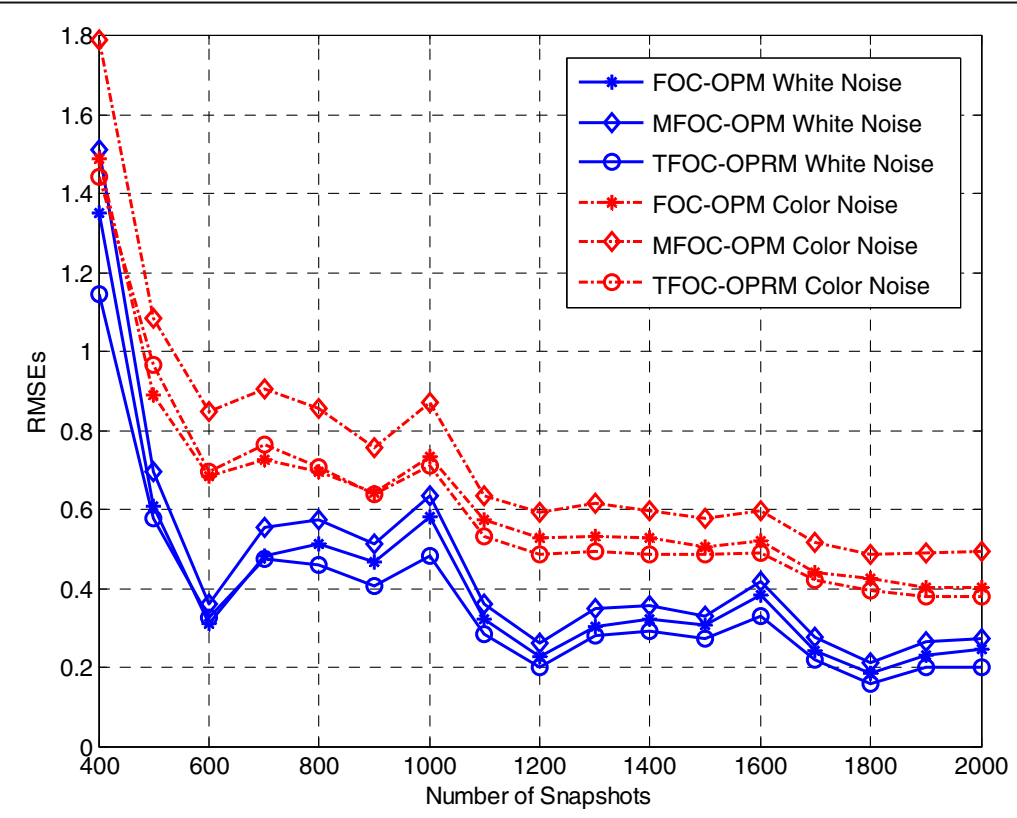

Fig. 4 RMSEs of the DOAs versus snapshots 


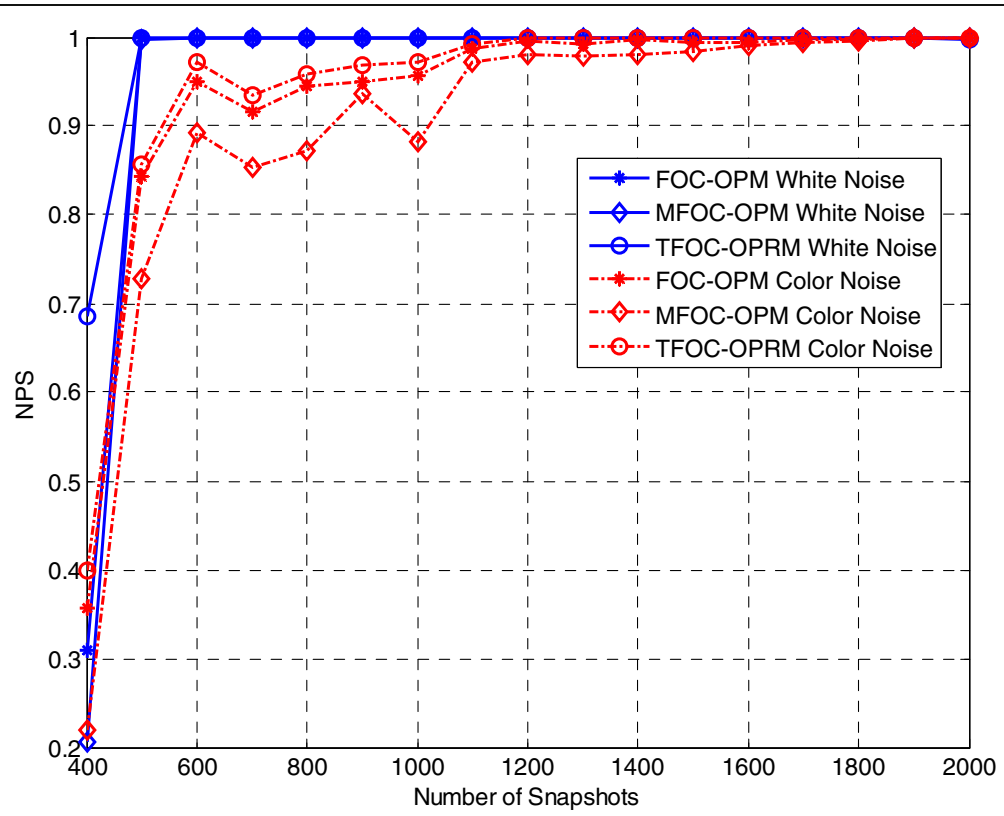

Fig. 5 NPS of the DOAs versus snapshots

mate method is performed on the reduced-rank $\mathbf{R}_{4}$ to improve estimation performance. Meanwhile, compared to MFOC-OPM method, the TFOC-OPRM algorithm has lower computational burden, which exploits polynomial rooting instead of spectral search.

\subsection{Experiment 4: the calculation complexity versus snapshots}

In this simulation experiment, we further verify the advantages of TFOC-OPRM algorithm in terms of computational complexity, also by comparing the algorithms with FOC - OPM and MFOC - OPM. The number of incident target signal sources and the number of array elements of ULA are set as $M=3$ and $N=3$ respectively, with the interval of angular scanning being defined as $\Delta \theta=0.01$. Figure 6 shows the calculation complexity of the proposed TFOC - OPRMalgorithm and the comparison algorithms as the number of sampling snapshots changes (the number of sampling snapshots changes from $L=400$ to $L=2000$ ). Viewing from the simulation results in Fig. 6, with the increasing number of sampling snapshots, the computational complexity of the proposed TFOC - OPRM algorithm is significantly far lower than that of the FOC-OPM algorithm and the MFOC - OPM algorithm, and this advantage will be more obvious with the further increase of the number of sampling snapshots. The reason is that the proposed TFOC - OPRM algorithm not only eliminates a large number of redundant data in the original FOC, but also adopts the polynomial root method. This is consistent with the theoretical analysis given in Section 3.3 and testify the high-efficiency of the proposed TFOC - OPRM algorithm.

\section{Conclusions}

In this paper, a novel low computational complexity TFOC - OPRM localization algorithm for DOA estimation has been proposed in the presence of spatially white noise 


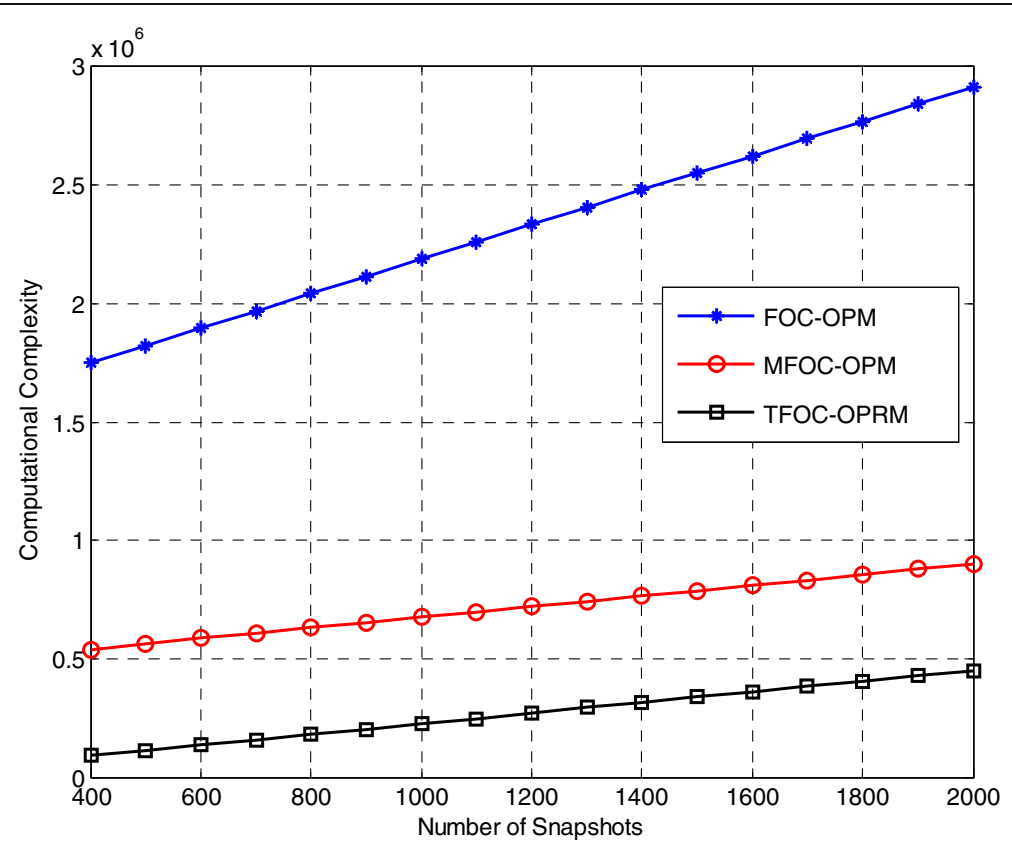

Fig. 6 Computational complexity comparison versus the number of snapshots

and spatially color noise environments. Specifically, we reconstruct a new Toeplitz matrix, which is close to the Toeplitz structure information in ideal condition via the Toeplitz approximate method. By exploiting the polynomial root method, the proposed TFOC - OPRM localization algorithm does not include large amount of EVD or SVD computation load, which are required in conventional DOA estimation algorithms such as the MUSIC or ESPRIT algorithms. All simulation results validate the superiority of the proposed TFOC-OPRM localization algorithm. Moreover, the simulation results indicate that the proposed TFOC - OPRM localization algorithm achieves lower computational complexity and better accuracy than the FOC-OPM algorithm and the MFOC-OPM algorithm both in spatially white noise and spatially color noise situations.

\section{Abbreviations}

FOC: fourth-order cumulants; TFOC-OPRM: Toeplitz fourth-order cumulants orthonormal propagator rooting method; DOA: direction-of-arrival; ULA: uniform linear array; MFOC: modified fourth-order cumulants; TFOC: Toeplitz fourthorder cumulants; OPM: orthonormal propagator method; MUSIC: multiple signal classification algorithm; ESPR IT: estimating signal parameter via rotational invariance techniques; DOAs: direction-of-arrivals; HOC: high-order cumulants; EVD: eigenvalue decomposition; SVD: singular value decomposition; SNR: signal-to-noise ratio; FOCOPM: fourth-order cumulants orthonormal propagator method; MFOC-OPM: modified fourth-order cumulants fourthorder cumulants; NPS: normalized probability of success; RMSEs: root-mean-square-errors

\section{Acknowledgements}

The authors acknowledged the anonymous reviewers and editors for their efforts in valuable comments and suggestions.

\section{Authors' contributions}

H.P.S proposed the main idea, designed the experiments, and discussed the results. N.M and Z.W.G wrote the paper. L.Z.Z, and S.J gave some important suggestions and revised the paper. All authors read and approved the final manuscript. 
Research Program of Tianjin Municipal Education Committee under Grant No. JWK1609, and by the Artificial intelligence Science and Technology Support planning Major project of Tianjin under Grant No. 17ZXRGGX00070.

Availability of data and materials

The datasets used and/or analyzed during the current study are available from the corresponding author on reasonable request.

\section{Ethics approval and consent to participate}

This article does not contain any studies with human participants or animals performed by any of the authors.

\section{Competing interests}

The authors declare that they have no competing interests.

\section{Author details}

${ }^{1}$ School of Automobile and Transportation, Tianjin University of Technology and Education, Tianjin 300222, China. ${ }^{2}$ School of Electronic Engineering, Tianjin University of Technology and Education, Tianjin 300222, China. ${ }^{3}$ Tianjin Sino-German University of Applied Sciences, Tianjin 300350, China. ${ }^{4}$ CATARC Testing and Certification Business Division, China Automotive Technology and Research Center Co., Ltd, Tianjin 300300, China.

Received: 2 June 2020 Accepted: 17 September 2020

Published online: 07 October 2020

\section{References}

1. H. Krim, M. Viberg, Two decades of array signal processing research: The parametric approach. IEEE Signal Process. Mag. 13(4), 67-94 (1996)

2. F. Wen, Computationally efficient DOA estimation algorithm for MIMO radar with imperfect waveforms. IEEE Commun. Lett. 23(6), 1037-1040 (2019)

3. E. Claudio, R. Parisi, G. Jacovitti, Space time MUSIC: Consistent signal subspace estimation for wideband sensor arrays. IEEE Trans. Signal Process. 66(10), 2685-2699 (2018)

4. X. Dai, X. Zhang, Y. Wang, Extended DOA-matrix method for DOA estimation via two parallel linear arrays. IEEE Commun. Lett. 23(11), 1981-1984 (2019)

5. R. Schmidt, Multiple emitter location and signal parameter estimation. IEEE Trans. Antennas Propag. 34(3), 276-280 (1986)

6. R. Roy, T. Kailath, ESPRIT-estimation of signal parameters via rotational invariance techniques. IEEE Trans. Acoust. Speech Signal Process. 37(7), 984-995 (1989)

7. Z. Shan, T.P. Yum, A conjugate augmented approach to direction-of-arrival estimation. IEEE Trans. Signal Process. 53(11), 4104-4109 (2005)

8. W. Zeng, X. Li, X. Zhang, Direction-of-arrival estimation based on the joint diagonalization structure of multiple fourthorder cumulant matrices. IEEE Signal Processing Letters 16(3), 164-167 (2009)

9. J. He, Z. Zhang, C. Gu, T. Shu, W. Yu, Cumulant-based 2-D direction estimation using an acoustic vector sensor array. IEEE Trans. Aerosp. Electron. Syst. 56(2), 956-971 (2020)

10. B. Liao, S. Chan, A cumulant-based method for direction finding in uniform linear arrays with mutual coupling. IEEE Antennas Wireless Propagation Letters 13, 1717, 2014-1720

11. P. Chevalier, L. Albera, A. Ferreol, P. Comon, On the virtual array concept for higher order array processing. IEEE Trans. Signal Process. 53(4), 1254-1271 (2005)

12. S. Marcos, A. Marsal and M. Benidir, "Performances analysis of the propagator method for source bearing estimation," IEEE International Conference on Acoustics, Speech and Signal Processing (ICASSP), Adelaide, SA, 1994

13. S. Marcos, A. Marsal, M. Benidir, The propagator method for source bearing estimation. Signal Process. 42(2), 121-138 (1995)

14. L. An and W. Shu, "Propagator method for DOA estimation using fourth-order cumulant," 2011 7th International Conference on Wireless Communications, Networking and Mobile Computing (WiCOM), Wuhan, 1-4, 2011.

15. J. He, Z. Liu, Extended aperture 2-D direction finding with a two-parallel-shape-array using propagator method. IEEE Antennas Wireless Propagation Letters 8, 323-327 (2009)

16. T. Ahmed, X. Zhang, W.U. Hassan, A higher-order propagator method for 2D-DOA estimation in massive MIMO systems. IEEE Commun. Lett. 24(3), 543-547 (2020)

17. P. Palanisamy, N. Rao, Direction of arrival estimation based on fourth-order cumulant using propagator method. Prog Electromagnetics Res B 18, 83-99 (2009)

18. J. Tang, X. Si, Q. Peng, Estimation of signal parameters via rotational invariance techniques algorithm with speedy fourth-order cumulants. J. Xi'an Jiaotong Univ. 43(6), 88-92 (2009)

19. D. Zhang, Y. Zhang, G. Zheng, C. Feng, J. Tang, Improved DOA estimation algorithm for co-prime linear arrays using root-MUSIC algorithm. Electron. Lett. 53(18), 1277-1279 (2017)

20. J. Selva, Computation of spectral and root MUSIC through real polynomial rooting. IEEE Trans. Signal Process. 53(5), 1923-1927 (2005)

21. X. Meng, J. Xue, F. Yan, X. Yan, Real-valued propagator method for fast DOA estimation via polynomial rooting. J Eng 2019(21), 7792-7795 (2019)

22. F. Yan, X. Li, T. Jin, L. Liu, M. Jin, A real-valued polynomial rooting method for fast direction of arrival estimation with large uniform linear arrays. IEEE Access 7, 122330-122341 (2019)

23. S.R. Best, A discussion on the significance of geometry in determining the resonant behavior of fractal and other nonEuclidean wire antennas. IEEE Antennas Propagation Magazine 45(3), 9-28 (2003)

24. E. Guariglia, Entropy and fractal antennas. Entropy 18(3), 84 (2016) 
25. S.R. Best, Operating band comparison of the perturbed Sierpinski and modified Parany gasket antennas. IEEE Antennas Wireless Propagation Letters 1, 35-38 (2002)

26. E. Guariglia, Harmonic Sierpinski gasket and applications. Entropy 20(9), 714 (2018)

27. W.J. Krzysztofik, Fractal geometry in electromagnetics applications-from antenna to metamaterials. Microwave Rev 19(2), 3-14 (2013)

28. R.G. Hohlfeld, N. Cohen, Self-similarity and the geometric requirements for frequency independence in antennae. Fractals-complex Geometry Patterns \& Scaling in Nature \& Society 7(01), 79-84 (1999)

29. E. Guariglia, "Spectral analysis of the Weierstrass-Mandelbrot function," 2017 2nd International Multidisciplinary Conference on Computer and Energy Science (SpliTech), Split, pp. 1-6, 2017.

30. E. Guariglia, S. Silvestrov, in Engineering Mathematics II, Springer Proceedings in Mathematics \& Statistics, ed. by S. Silvestrov, M. Rančić. Fractional-wavelet analysis of positive definite distributions and wavelets on D'(C), vol 179 (2016), pp. 337-353

31. P. Chevalier, A. Ferreol, On the virtual array concept for the fourth-order direction finding problem. IEEE Trans. Signal Process. 47(9), 2592-2595 (1999)

32. A. J. Barabell, "Improving the resolution performance of eigen-structure-based direction-finding algorithms," Proc. IEEE Int. Conf. Acoust., Speech, Signal Process., Boston, MA, USA, May, 336-339, 1983.

33. B. Rao, K. Hari, Performance analysis of root-MUSIC. IEEE Trans. Acoust. Speech Signal Process. 37(12), 1939-1949 (1989)

34. Q. Ren, A. Willis, Fast root MUSIC algorithm. Electron. Lett. 33(6), 450-451 (1997)

\section{Publisher's Note}

Springer Nature remains neutral with regard to jurisdictional claims in published maps and institutional affiliations.

\section{Submit your manuscript to a SpringerOpen ${ }^{\circ}$} journal and benefit from:

- Convenient online submission

$\checkmark$ Rigorous peer review

- Open access: articles freely available online

High visibility within the field

- Retaining the copyright to your article 